
allemande

45-2 | 2013

Images et discours de la nation

\title{
Memory-Effekt. Überlegungen zur künstlichen Erinnerung
}

Michael Lück

\section{(2) OpenEdition}

1 Journals

Édition électronique

URL : https://journals.openedition.org/allemagne/1523

DOI : 10.4000/allemagne. 1523

ISSN : 2605-7913

Éditeur

Société d'études allemandes

Édition imprimée

Date de publication : 30 décembre 2013

Pagination : 357-368

ISSN : 0035-0974

\section{Référence électronique}

Michael Lück, „Memory-Effekt. Überlegungen zur künstlichen Erinnerung", Revue d'Allemagne et des pays de langue allemande [Online], 45-2 | 2013, Online erschienen am: 29 Juli 2019, abgerufen am 22 Mai 2021. URL: http://journals.openedition.org/allemagne/1523 ; DOI: https://doi.org/10.4000/ allemagne.1523 


\section{Memory-Effekt. Überlegungen zur künstlichen Erinnerung}

- Michael Lück*

„Ich, Jonathan Meese, die Ameise der Kunst, habe mit Adolf Hitler kein Problem, tut mir leid - vor allen Dingen nicht mit den Abbildungen. In einer Fotografie von Adolf Hitler ist Adolf Hitler gar nicht mehr drin, und in einem Picasso-Bild ist auch Picasso nicht drin. Den kann ich nicht rausdestillieren. Ich kann das Bild nicht zerquetschen und dann kommt Picasso raus oder Adolf Hitler. Is' ein Bild, ein Abbild, ein Foto."

Jonathan Meese, Kommentar in der Dokumentation „Jonathan Meese - Eine Ameise der Kunst“, ARD 2008.

\section{Geister}

Im August 2004 erscheint auf dem Cover des deutschen Nachrichtenmagazins Der Spiegel das Konterfei Adolf Hitlers (Abb. 1). Das war längst nicht ungewöhnlich. Zum ersten Mal blickte Hitler im Frühjahr 1964 aus dem roten Wechselrahmen des Spiegels, und bis zum heutigen Tag sind 47 weitere Hitler-Titelbilder hinzugekommen - wobei 31 davon aus den letzten beiden Jahrzehnten stammen ${ }^{(1)}$. Das ist nicht bloß Erbsenzählerei. Es scheint fast, als leuchte die Physiognomie Hitlers ständig und immer deutlicher im Display des Magazins nach - eine Art Image Persistance wie etwa beim sogenannten LCD-Memory-Effekt, bei dem nach der permanenten Anzeige eines Bilds die durch Spannung erzeugte Lichtdurchlässigkeit der Flüssigkristalle vor allem in hellen Bildpunkten bestehen bleibt, auch wenn die Anregungsspannung, also das aktuelle Bild sich verändert ${ }^{(2)}$. Es kommt zu einer Geisterbild-Störung.

* M. A., Freie Universität Berlin.

1 Vgl. http://www.umblaetterer.de/2010/11/07/hitler-titel-des-spiegel/; http://spiegelstudien.de; Simone ERPEL, „Hitler entdämonisiert. Die mediale Präsenz des Diktators nach 1945 in Presse und Internet“, in: Deutsches Historisches Museum (Hg.), Hitler und die Deutschen. Volksgemeinschaft und Verbrechen (Katalog zur gleichnamigen Ausstellung, DHM Berlin, 15.10.2010 bis 27.2.2011), Dresden, 2010, S. 154-160.

2 Eine andere Image Persistance stellt sich beim Plasmabildschirm ein, wenn die Phosphor-Leuchtstoffe, die im Kontakt mit ionisiertem Gas (Plasma) Licht emittieren, ihre Luminanz durch helle „Dauerleuchtung" einbüßen. 
Auch im Durchgang der Hitler-Titelbilder scheint sich eine Art Persistenz einzustellen, die über das aktuelle Cover hinausgeht und sich dabei ablöst von den jeweiligen Titelstories. Freilich erstreckt sich die Abfolge der Hitler-Bilder des Spiegels, wenngleich beschleunigt seit den 80ern, über Jahrzehnte. Und das Image „Zwischen“ oder „unter“ den Bildern bleibt eine Art Metapher für einen diskursiven Effekt, sofern und solange das Intervall der Bilder angefüllt ist mit dem Reden und Schreiben über Vergangenheit.

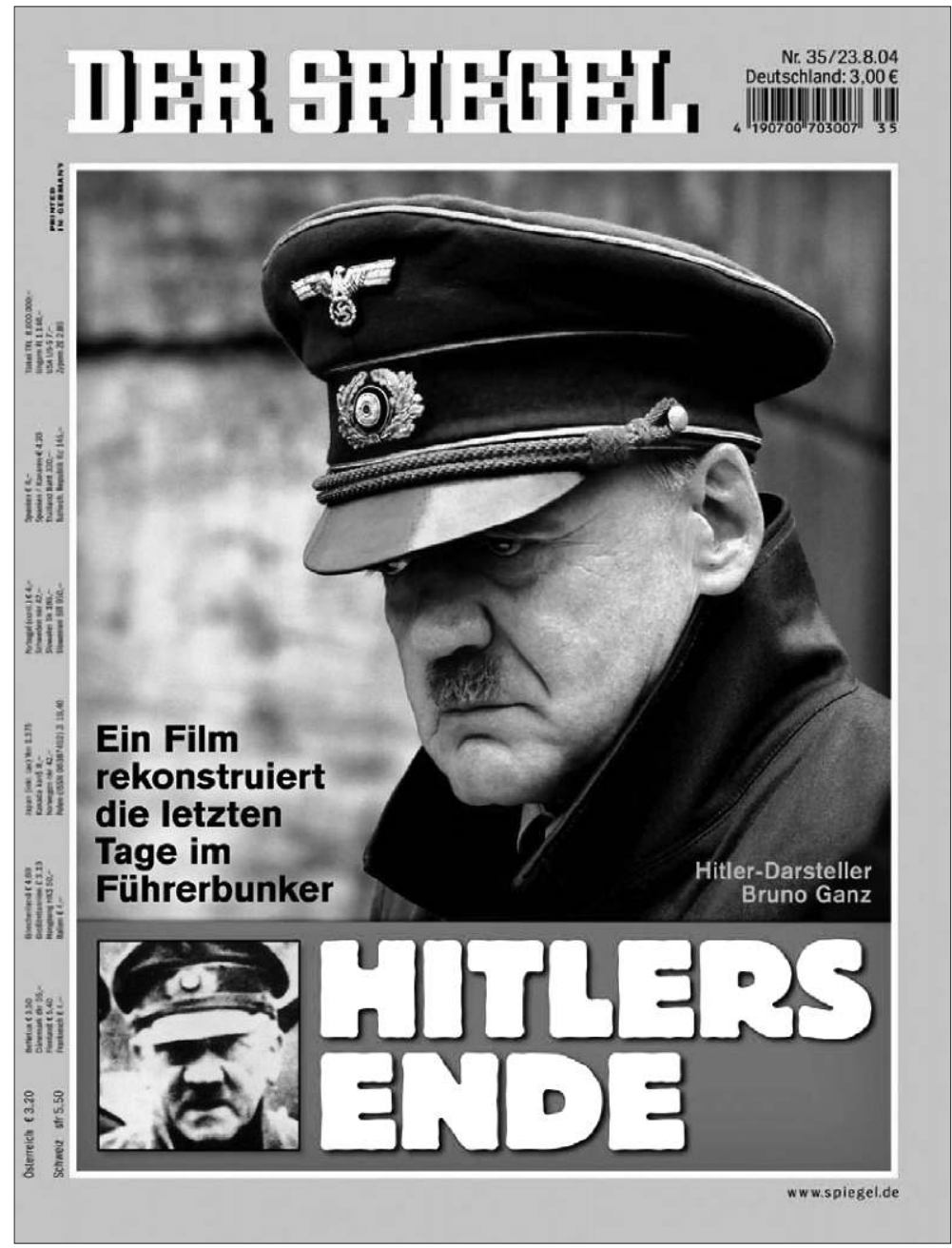

Der Spiegel 35/2004

\section{Treiben im Bildfeld}

Noch einmal das Spiegel-Cover vom August 2004. Das Entscheidende an diesem Bild blieb bislang unerwähnt. Wir sehen den schweizerischen Schauspieler Bruno Ganz in der Rolle Adolf Hitlers. Es handelt sich um ein Bild aus dem Film Der Untergang von Oliver Hirschbiegel, der am 9. September 2004 in München uraufgeführt wurde. Hitler 
als Rolle, als Hauptrolle: für sich genommen ist dieser Umstand 2004 ebenso wenig ungewöhnlich wie ein Hitler-Titelbild des Spiegels. Hirschbiegels Projekt hatte zumindest in dieser Hinsicht mit Filmen wie Der letzte Akt von Georg Wilhelm Pabst aus dem Jahr 1955 (mit Albin Skoda als Adolf Hitler) oder The Bunker von George Schaefer aus dem Jahr 1981 (mit Anthony Hopkins in der Hitler-Rolle) unmittelbare Vorläufer. Was nun auf dem Cover von 2004 geschieht, ist, verkürzt gesprochen, dass der Filmframe mit der Bilderkolonne des Nachrichtenmagazins, die Leinwand mit der Titelseite verschaltet wird, ohne dass eines von beidem ganz aufgehoben würde. Diese Verschaltung findet zunächst dadurch statt, dass das Filmbild, ausgerichtet am unverwechselbaren roten Rahmen des Magazins, genau den Platz besetzt, den bis dahin die Hitler-Collagen, -Zeichnungen oder Archivbilder eingenommen hatten. Darüber hinaus wird diese Umsetzung selbst noch einmal ins Bild hineingeholt. Das ausgewechselte Format des reproduzierten Archivmaterials bleibt im aktuellen Titelbild erhalten, es ist ins linke untere Bildfeld abgerückt worden und steht im Spannungsverhältnis zur Aufnahme im Zentrum. Das fotografische Dokument, schwarz-weiß, zeigt einen direkten Blick Hitlers in die Kamera. Dieser Blick jedoch lässt sich nicht annähern. Die offensichtlich nachträglich vorgenommene Ausschnittvergrößerung lässt die Fotografie nur noch mehr verrauschen, sodass sich der ein für allemal zeitlich und räumlich fixierte, also historische Platz der Kamera als eine Art Bannkreis bemerkbar zu machen scheint. Der Betrachter bleibt an den historischen Platz und das eingeschriebene Distanzverhältnis gebunden. Demgegenüber weist das farbige, desaturierte Filmbild kein sichtbares Rauschen auf. Der Blick der Hitler-Figur ist nach innen gerichtet. Das Gesicht im Halbprofil wirkt nah. Die geringe Schärfentiefe der langen Brennweite, der verflachte Raum, lässt das Bild einen Blick realisieren, der, wie von ihm „unbemerkt“, die Distanz zum Angeblickten, zu Hitler, überspringen konnte. Die Umrissklarheit und Nähewirkung lässt sich jedoch nicht allein auf die Tatsache einer Brennweite zurückrechnen, sie setzt in gewisser Weise auch das andere Bild, das fotografische Dokument voraus. Sie besteht in der Relation, wie sie die Titelseite insgesamt bildet. Die Nähe ist auch die Aufhebung der Distanz in der veränderten Bildräumlichkeit sowie die Aufspaltung der Blickachse in einen versunkenen Blick einerseits und dessen Beobachtung andererseits. Das eine Bild bleibt so im andern enthalten. Diese Anwesenheit in der Unterschiedenheit zeigt sich ganz deutlich. Wir sehen dieselbe Physiognomie: hier wie dort der fallende Mundwinkel, die tiefe Nasolabialfalte, die knollige Nase, die Verschattung der Augenpartie. Und dennoch handelt es sich nicht um eine Reproduktion, um eine Nachstellung dieses einen, ganz bestimmten dokumentarischen Bilds mit seinen spezifischen historischen Informationen, von denen der Zuschnitt auch gar nicht mehr viel übriggelassen hat. Die Menge der Bilder, die an der physiognomischen Gestalt „Hitler" teilhaben, ist, anders als die der zumindest theoretisch abzählbaren historischen Zeugnisse, unbestimmt. An die Stelle des einen fotografischen Dokuments am Rand des Spiegel-Covers ließen sich grundsätzlich nicht nur weitere zeitgenössische Fotografien, Gemälde, Skulpturen setzen, sondern auch spätere Zeichnungen, Karikaturen, Beschreibungen - oder Coverbilder. Zwar ist das Filmbild, das der Spiegel vom August 2004 zeigt, innerhalb des Filmverlaufs vermutlich narrativ auf ein historisches Ereignis bezogen, doch es referiert visuell nicht auf ein isolierbares historisches Einzelbild. Vielmehr ist es auf ein stets erweiterbares, ein virtuelles Bildfeld bezogen. Indem es seinerseits an der physiognomischen Gestalt 
teilhat, ohne ein historisches Bild nachzustellen, ohne also in einer Referenz verankert zu sein, aktiviert es dieses Bildfeld gerade in seiner Erweiterbarkeit. Es ist, wenn man so will: selbst ein Tropfen im Meer auf dem es treibt. Die Frage der „Quelle“ läuft damit ins Leere. Die Aktualität des Bilds besteht in der eigentümlichen Anwesenheit eines immer nur virtuellen Bildfelds, sie besteht nicht im Wiederbringen, im aktualisierenden Zugriff auf ein bestimmtes Bild.

Das Spiegel-Cover liefert damit eine recht präzise Analyse des inszenatorischen Grundmusters des Untergangs. Auch er erzeugt, ähnlich der Ausstellungswand im Deutschen Historischen Museum, ein Bildintervall, mit dem sich eine Gleichzeitigkeit gegen das Nacheinander der Bilder, gegen die Zeit des historischen Bezugs und der Auslegung verschiebt.

Der Film fängt an mit einem kurzen Ausschnitt aus der Dokumentation Im toten Winkel - Hitlers Sekretärin von André Heller aus dem Jahr 2002. Die Kamera ist unbewegt, das Licht wirkt hart, der Kontrastumfang ist gering und die Körnung des Filmmaterials scheint irgendwie grob. Man sieht ein Interview-Setting: Eine ältere Frau sitzt vor einer Bücherwand und redet, sie fokussiert dabei ein Gesprächsgegenüber, das nicht im Bild ist. Die Einstellung scheint bearbeitet, der Ausschnitt ist vergrößert, aufgezogen zu einer fürs dokumentarische Format ungewöhnlichen Nahaufnahme. Tatsächlich gibt es keinerlei Hinweis auf den Ursprung des Bilds, auf die Dokumentation, doch die Spuren der Bearbeitung lassen ganz deutlich den Eindruck entstehen, dass es, von irgendwo, hierher gestellt wurde. Der Film Der Untergang beginnt also, was die Bilder angeht, von vornherein mit einer Umstellung genau dort, wo es eine Referenz hätte geben können.

So weiß man zunächst auch nicht, wer die Frau ist. Erst später wird man darauf kommen, dass es sich um Gertrude Junge handelt, geborene Humps, Jahrgang 1920, Hitlers Sekretärin im sogenannten „Führerbunker“ unter der Reichskanzlei in Berlin. Man hört jetzt ihre Stimme im akustischen Vordergrund. Sie spricht von einem jungen Ding, von einem Mädchen, dem sie nicht verzeihen kann. Dann spricht sie nur noch von sich und man merkt: das junge Ding ist sie selbst in der dritten Person. Sie redet von einem Schicksal, das sie vorangetrieben habe, davon, dass sie keine begeisterte Nationalsozialistin gewesen sei. Dann versucht sie, diese scheinbar reflexhafte Apologetik wieder zurückzunehmen, indem sie noch einmal beteuert, es falle ihr schwer, sich selbst zu verzeihen. Und dann friert das Bild ein. Die Bewegung endet, bevor es zu einer Montageoperation, einem Schnitt oder einer Blende kommt. Die Bewegung im Bild und die Dauer des Bilds selbst treten auseinander. Die Verzögerung oder Dehnung des freeze frame hebt ihre Synchronisierung auf. Die ist jedoch entscheidend für den Eindruck eines Nacheinanders, einer Kontinuität. Das bewegte Bild erscheint hier also nicht mehr wie eine Folge der Bewegung im Bild, es behauptet eine eigene Zeit: die Dauer des Frames. Und noch einmal wiederholt sich in dieser Behauptung, in der Differenz von Bewegung und Zeit, der Eindruck, dass das Bild hierher gestellt wurde, dass es nicht identisch ist mit dem Film.

Erst jetzt, da die visuelle Bewegung so abrupt aufgehoben wird, stößt man mit den Ohren auf die Musik, deren Bewegung, also der Klang, sich ungehindert fortsetzt. Das Adagio aus stehenden Streichern und hineintropfenden Klavierklängen dominierte von Beginn an den audiovisuellen Zusammenhang, es hatte mit der Einblendung des Titels eingesetzt und war also der Stimme der Frau vorausgegangen. Der Raum, die Frequenz 
und die Dauer des Klangs realisierten sich in der Zuschauerwahrnehmung wie das akustische Medium des darauffolgenden Bilds, der Nahaufnahme Traudel Junges. Jetzt löst sich der Klang wieder von diesem Bild ab, und das nächste Bild stellt sich in ihm ein. Der freeze frame geht über in eine Schwarzblende. Die musikalische Dynamik nimmt zu. Dann hebt sich ein knisterndes Geräusch von der Musik ab. Dieses Geräusch wird gewissermaßen zum akustischen Ausgangspunkt des nächsten Bilds. Man sieht zunächst nur einen kleinen Lichtpunkt, so vereinzelt und flackernd wie das Geräusch eine Art synästhetische Homologie. In der Aufblende erweist sich der Lichtpunkt dann als Schein einer Taschenlampe in der Hand eines Soldaten. Schwaches Licht fällt auf ihn und auf eine Gruppe von Frauen, die sich aus einem tiefschwarzen Hintergrund auf die Kamera zu bewegen. Das knisternde Geräusch dehnt sich in der Bewegung der Aufblende aus, es wird in dieser Erweiterung gleichsam diegetisch, es wird auf das gegenständliche Bild gespannt und verändert sich dabei zur Kenntlichkeit: zum Rascheln von Laub. Als eine der Frauen (gespielt von Alexandra Maria Lara) sich ins Format einer Nahaufnahme hinein der Kamera genähert hat, wird ihr Gesicht durch eine neue helle Lichtquelle angestrahlt. Sie erschrickt wie von einem Blitzlicht überrascht.

Die gesamte Sequenz, von der Titeleinblendung bis zum Schlaglicht auf die Schauspielerin Alexandra Maria Lara, lässt sich nicht als eine Rückblende beschreiben. Zwar wird sie von zwei Einstellungen geklammert, die das Gesicht Traudel Junges mit dem der Schauspielerin verknüpfen, doch wirkt die erste dieser Einstellungen von vornherein wie versetzt. Sie verweist ihrerseits auf ein unausgemachtes Irgendwoher und schiebt sich zwangsläufig als Anfang oder Bezugspunkt der späteren Einstellung auf. Die Sequenz stellt kaum eine narrative Aktualisierung dar. Vielmehr entspricht sie einer musikdramaturgischen Operation. Tatsächlich bildet die Musik hier jene Zeitschicht, auf der die Bilder in ein Nebeneinander geraten. Es ist die Musik, mit der sich, abgelöst von der Bewegung im Bild, die Dauer des freeze frames verbindet. Als Klang realisiert sich diese Dauer sinnlich in der Zuschauerwahrnehmung, und im Klang geht sie schließlich auf. Und es ist die Musik, aus der heraus sich dann, wie beschrieben, die nächste Einstellung zunächst als Vergegenständlichung eines Knisterns entwickelt.

Es gibt keinen Nullpunkt der Erzählung in der Zeit der Musik, und sie trägt keine historische Signatur. Alles beginnt, wagnerianisch, aus dem unsichtbaren Orchestergraben ${ }^{(3)}$. So sind die Worte Traudel Junges, die sich zudem selbst in eine Erste und eine Dritte Person aufzulösen scheint, gleichermaßen als Erzählinstanz wie als historisches Zeugnis aufgehoben. Die Sequenz setzt demnach keinen autobiografischen Erzählmodus in eine audiovisuelle Struktur von Erinnertem um. Vielmehr bildet sie jene Art von Tableau vor, das die Inszenierung des Untergangs kennzeichnet.

In ihm realisiert sich keine Perspektive, die an ein erinnerndes, sich selbst historisch auslegendes Subjekt gebunden wäre. Wie das fotografische Dokument auf dem Spiegel-Cover vom August 2004 stellt der ebenfalls nachbearbeitete dokumentarische Ausschnitt zu Beginn des Films keinen Referenzpunkt dar. Er bringt das immer erweiterbare, virtuelle Feld der Bilder, Töne, Texte in Spiel, dessen Anwesenheit der Film

3 Vgl. hierzu Friedrich Kittler, „Weltatem. Über Wagners Medientechnologie“, in: Ders., Manfred Schneider, Samuel Weber (Hg.), Medien (Diskursanalysen 1), Opladen, Westdeutscher Verlag, 1987, S. 94-107. 
gebildet haben wird - eben jene Art Anwesenheit, wie sie für die Anfangssequenz allein in der Zeit der Musik zwischen der eingefrorenen Nahaufnahme Traudel Junges und der Schockstarre im Gesicht Alexandra Maria Laras besteht.

$\mathrm{Zu}$ den Bildern, Tönen, Texten im virtuellen Feld gehört Traudel Junges Zeugnis, das sie kurz nach Kriegsende verfasste, ebenso wie die Aussagen, die der amerikanische Richter Michael Angelo Musmanno im Rahmen der Nürnberger Nachfolgeprozesse sammelte und 1950 unter dem Titel Ten Days to Die herausgab ${ }^{(4)}$. Das Buch Der Untergang des Hitler- und Albers Speer-Biografen Joachim Fest gehört dazu und die Artikel des Spiegels aus den 1990er Jahren, die vom Berliner „Führerbunker“, von den „letzen Tagen“ des „Tausendjährigen Reichs“ und vom Verbleib der Hitlerleiche berichten. Ihr Bildmaterial, die Skizzen und Grafiken wirken fast wie das storyboard des Films ${ }^{(5)}$. Und es gehören auch die Archivbilder dazu, wie sie problemlos über youtube abgerufen werden können - etwa die Aufnahmen der 755. Deutschen Wochenschau vom März $1945^{(6)}$. In der Gegenüberstellung von Abb. 2 wirken diese Aufnahmen zunächst wie das Ausgangsmaterial der Filmbilder. Man sieht eine Szene aus dem ersten Drittel des Films: Hitler steigt aus dem Bunker nahe der Reichskanzlei und verleiht Orden an die Berliner „Hitler Jugend“. Und dennoch geht das, was man in dieser Szene hört und sieht, nicht auf in einer Aktualisierung der Bilder der letzten Deutschen Wochenschau, die von diesem Ereignis berichten. Die Instabilität und das Schwanken des Bildrahmens, Kennzeichen einer Handkamera, setzt sich von der Reinszenierung der Eigenschaften des historischen Bilds ab ${ }^{(7)}$. Die Handkamera erscheint selbst im verwackelten Bild. Unabhängig von jeder handlungslogischen Plausibilisierung ihres Erscheinens hört sie damit für die Zeit des Bilds auf, die Voraussetzung oder der Ausgangspunkt eben dieses Bilds und seiner Eigenschaften zu sein. Das Bild selbst verschiebt diese Eigenschaften gegen einen historischen Ursprung und setzt sie gewissermaßen frei. So können sie sich einerseits sehr deutlich mit dem Schauspiel Bruno Granz verbinden, mit dem Schwanken und Zittern, dem Leiden der Hitlerfigur. Andererseits erzeugen sie zugleich eben jene Virtualität, in der sich der Platz des einen Wochenschau-Bilds zu einem unabschließbaren Feld erweitert.

In diesem Feld liegen auch die Beschreibungen des „Untergang[s] der Reichshauptstadt in drei Akten“, wie sie etwa Theodor Plivier in seinem Roman Berlin aus dem Jahr 1954 vorgelegt hat ${ }^{(8)}$. Anwesend sind solche literarischen Stadtbilder nicht als Nachstellung eines bestimmten Texts, sondern, wie hier, als Hall, im Geräusch des entfernten

4 Traudel Junge, Melissa MüLler, Bis zur letzten Stunde. Hitlers Sekretärin erzählt ihr Leben, Berlin, Claassen verlag, 2004; Michael A. Musmanno, In zehn Tagen kommt der Tod, München, Droemer, 1950.

5 Joachim C. Fest, Der Untergang. Hitler und das Ende des Dritten Reiches, Berlin, Alexander Fest, 2002; Anonymus, „Hitlers letzte Reise. Neue Thesen des Moskauer Historiker Lew Besymenski über den Tod des Führers und den Verbleib der Leiche“, Der Spiegel, 32 (1992); Anonymus, „Hitlers Höllenfahrt“, Der Spiegel, 14 (1995); AnONY mus, „Hitlers Höllenfahrt II“, Der Spiegel, 15 (1995).

6 http://www.youtube.com/watch?v=pEEHopx7Qpg.

7 Vgl. zum Re-enactment Robert Burgoyne, „Introduction: re-enactment and imagination in the historical film“, Leidschrift, 24 (2009), H. 3, S. 7-18.

8 Manuel Köppen, „Die letzten Tage des Reiches: Von Theodor Plieviers Roman Berlin zu Oliver Hirschbiegels Film Der Untergang“, in: Lars Koch, Marianne Vogel (Hg.), Imaginäre Welten im Widerstreit. Krieg und Geschichte in der deutschsprachigen Literatur seit 1900, Würzburg, Königshausen und Neumann, 2007, S. 304-319, 306. 


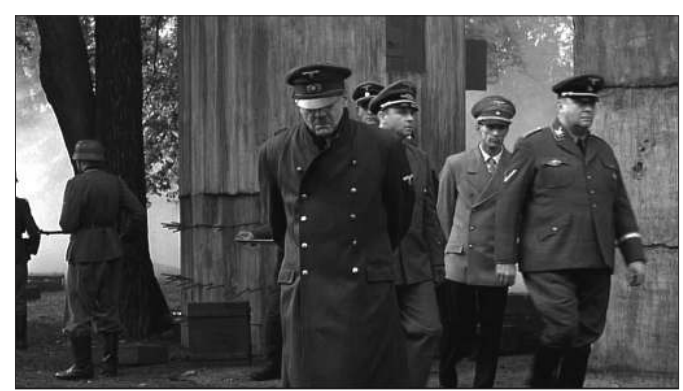

2.1: Der Untergang, D 2004, Oliver Hirschbiegel

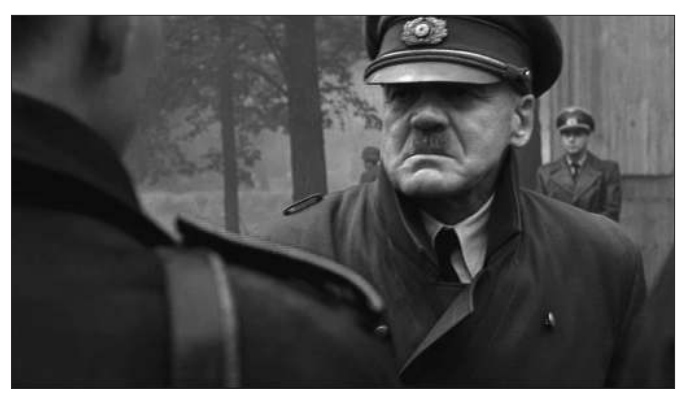

2.3: Der Untergang, D 2004, Oliver Hirschbiegel

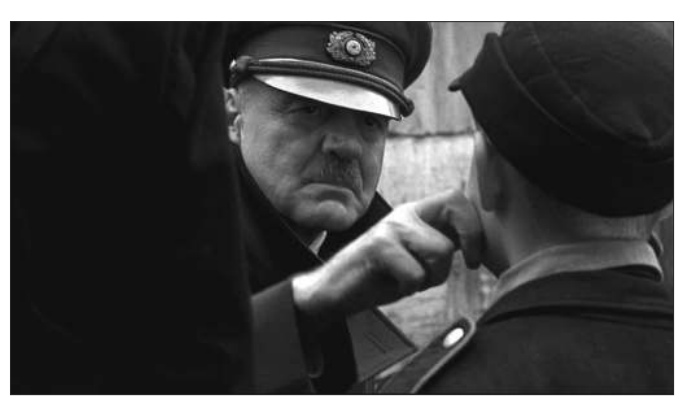

2.5: Der Untergang, D 2004, Oliver Hirschbiegel

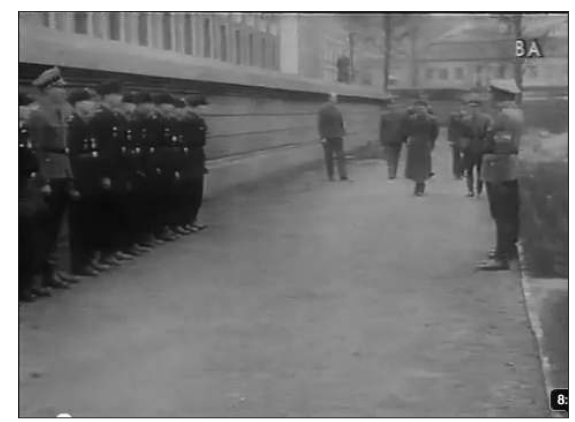

2.2: 755. Deutsche Wochenschau, 22. März 1945

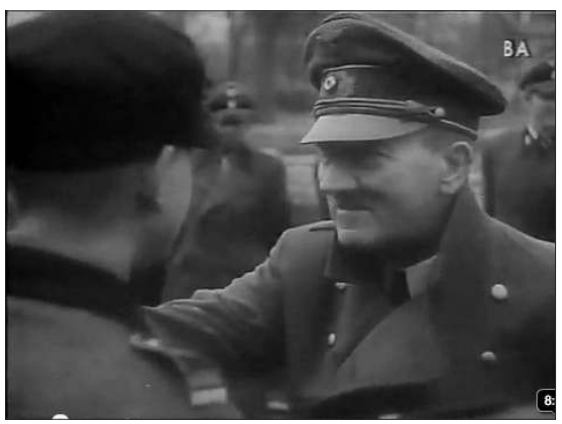

2.4: 755. Deutsche Wochenschau, 22. März 1945

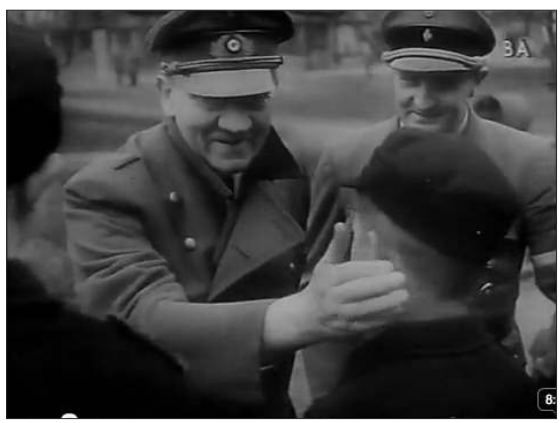

2.6: 755. Deutsche Wochenschau, 22. März 1945

Geschützlärms, der die Szene in ihrer gesamten Dauer überwölbt. Der akustische Raum macht die Stadtbeschreibung sinnlich anwesend, ohne ein bestimmtes Bild zu aktualisieren. Er ist in ähnlicher Weise gegen einen literarischen Ursprung verschoben, wie die instabile Kadrierung gegen eine historische Bildquelle. Wie sie erzeugt er ein virtuelles Feld und verbindet sich, als dessen Resonanzraum, zugleich mit dem Schauspiel.

In dieser Verbindung bildet die Szene ein Ausdrucksgefüge, das nicht als Auslegung eines bestimmten Bilds und seiner historischen, etwa propagandistischen Funktion oder eines bestimmten Texts verankert ist. Und es ist die Verlaufsform dieses treibenden Ausdrucksgefüges, nicht die dokumentierende oder fiktionalisierende Nacherzählung längstens bekannter historischer Ereignisse, die die Dramaturgie des Films Der Untergang prägt. Am Schauspiel Bruno Ganz entfaltet sich dieses Gefüge am deutlichsten. 
Das heißt aber auch, dass dieses Schauspiel, selbst gewissermaßen ursprungslos, ans virtuelle Feld verweist. Es lässt sich auf keinerlei symbolische Beziehung festlegen, etwa in dem Sinne, dass die Darstellung von Hitlers Gebrechlichkeit in einem Verhältnis stehe zu einem bestimmten Stadtbild, d.h. Kriegszustand Berlins und des Deutschen Reichs oder dass sie der Wirklichkeit, der Person hinter den Propagandabildern entspreche ${ }^{(9)}$. Vielmehr kann sich das viel beachtete Schauspiel nur genau in dem Maße so sehr entfalten, wie es eben keine historische Auslegung mehr ist.

Wenn Klaus Theweleit schreibt: „Je besser Bruno Ganz spielt, desto schlimmer“, registriert er kritisch genau diese Spannung zwischen ursprungslosem Ausdrucksgefüge und historischem Urteil, mit anderen Worten: die geisterhafte Gestalt des Films ${ }^{(10)}$. Mit Blick auf das, was derzeit in den Begriffen Erinnerungskultur und Mediengedächtnis beschrieben wird, zeigt sich, dass die Möglichkeit dieser Spannung nicht erst durch den Film selbst hergestellt wird und in welchem Verhältnis sie zum Umstand historischer Identität steht.

\section{Künstliche Erinnerung}

Filme wie Der Untergang, Ausstellungen wie Hitler und die Deutschen lassen sich, zumindest probeweise, auf etwas beziehen, wofür seit den 1990er Jahren der Begriff „Erinnerungskultur“ geprägt worden ist ${ }^{(11)}$. Die Erinnerungskultur umfasst, nach einem Vorschlag von Christoph Cornelißen, „alle denkbaren Formen der bewussten Erinnerung an historische Ereignisse, Persönlichkeiten und Prozesse [...], seien sie ästhetischer, politischer oder kognitiver Natur“ (12). Darüber hinaus bezeichnet das Stichwort jedoch auch einen spezifischen historischen Umstand. Demnach sind es Formen des Erinnerns, als die sich das politische, historische und auch das ästhetische Wahrnehmen und Urteilen nach dem Zweiten Weltkrieg zu realisieren beginnt. Die Musealisierung, also genau das, was seit 1987 im Deutschen Historischen Museum geschieht, wird beispielsweise in diesem Zusammenhang diskutiert ${ }^{(13)}$. Zugleich verzeichnet das

9 „Hitler als Mensch“ wurde zum Topos der Diskussion des Films in der deutschen Presse. Vgl. bspw. Andreas Kilb, „Die Ungeheuer mit dem menschlichen Zug. Der „Untergang“ und andere deutsche Filme in Australien“, Frankfurter Allgemeine Zeitung, 12.05.2005; Peter BECKER, „Erfolg des ,Untergangs‘. Mensch Hitler“, Der Tagesspiegel, 17.10.2004; Josef FrüCHTL, „Hitler als einer von uns. Menschsein im ,Untergang““, Frankfurter Rundschau, 24.09.2004; Harald WEnZEL, „Der erratische Führer. In ,Der Untergang“ wird Hitler zum tragischen Helden“, Frankfurter Rundschau, 18.09.2004.

10 „Chronik der laufenden Untergänge. Klaus Theweleit über das ,Hitler-Phänomen`, politischen Revisionismus, die Guido-Knopp-Ästhetik und über einen Film, den er nicht gesehen hat. Gespräch zwischen Klaus Theweleit und Michael Girke“, Freitag, Nr. 46, 5.11.2004. Vgl. auch Klaus ThewEleit, „,Der Untergang“ und andere Katastrophen. Gespräch mit Michael Girke“, in: Ders., Friendly Fire. Deadline-Texte, Frankfurt am Main/Basel, Stroemfeld, 2005, S. 389-409.

11 Aleida Assmann, Der lange Schatten der Vergangenheit. Erinnerungskultur und Geschichtspolitik, München, C. H. Beck, 2006; Christoph Cornelissen, „Was heißt Erinnerungskultur? Begriff - Methoden - Perspektiven“, Geschichte in Wissenschaft und Unterricht, 54 (2003), S. 548-563.

12 Christoph Cornelissen, „Erinnerungskulturen, Version:2.0“, Docupedia-Zeitgeschichte, 22.10.2012, URL: https://docupedia.de/zg/Erinnerungskulturen_Version_2.0_Christoph_Corneli.C3.9Fen?oldid=84892.

13 Vgl. Wolfgang Zacharias, „Zeitphänomen Musealisierung“, in: Ders. (Hg.), Zeitphänomen Musealisierung. Das Verschwinden der Gegenwart und die Konstitution der Erinnerung, Essen, Klartext, 1990, S. 9-30. Vgl. zur Kontroverse um das DHM, die Teil des sog. „Historikerstreits“ wurde, Christop Sтӧlzl (Hg.), Deutsches Historisches Museum. Ideen - Kontroversen - Perspektiven, Berlin (West), 
Stichwort den Eingang des Begriffs „Erinnerung“ in die historische Forschung, wo er einen erfahrungsgeschichtlichen Zugriff auf das Verhältnis zwischen Identitäten und der politischen Gemeinschaft der Nation ermöglichen soll ${ }^{(14)}$ - auch und gerade übers Zeitalter der europäischen Nationalstaatsbildung hinaus. Denn eben auch das registriert das Stichwort der Erinnerungskultur: eine Globalisierung der Erinnerung nach $1945^{(15)}$. Am Ungeheuren des Holocaust, des millionenfachen Mords an den europäischen Juden, bietet die Vergegenwärtigung der eigenen Nationalgeschichten, der politischen, sozialen, wirtschaftlichen, kulturellen Strukturen, keine ausreichende Orientierung mehr. Obgleich ein Ereignis in der Geschichte, erweist sich der Holocaust in diesem Sinne als historisch uneinholbar, als singulär. Aus eben diesem Grund lässt er sich auch über zeitliche und räumliche Grenzen, über Generationen und Staaten hinweg übertragen, sodass grundsätzlich überall auf der Welt Holocaustgedenkstätten entstehen können und die Erklärung, dass sich Auschwitz niemals wiederholen dürfe, nicht notwendig mit der Verantwortung vor der spezifischen Geschichte des eigenen Landes begründet wird, sondern mit der Verpflichtung gegenüber der universellen Menschenwürde.

Der Holocaust verweist durch seine historische Unbestimmbarkeit hindurch als Grenzfall von Nationalgeschichte offensichtlich auf eine andere Geschichte, eine Menschlichkeitsgeschichte, wenn man so will. Vor ihrem Hintergrund erst wird er als das historische Ereignis kenntlich, das er zweifellos ist. Der Begriff der Erinnerung bezeichnet ganz einfach den anderen Zugriff, den diese spezifische historische Ereignishaftigkeit verlangt.

In dieser Ereignishaftigkeit ist das Universelle unmittelbar mit dem Individuellen verknüpft, das Globale der Menschlichkeitsgeschichte mit einzelnen Handlungen, die in einer politischen System-, einer Sozial- oder Gesellschaftsgeschichte kaum abzubilden wären. Das Überschreiten der Nationalgeschichten durch das gleichwohl historische Ereignis des Holocaust lässt, nach einer Überlegung Wolfgang Hardtwigs, eine andere Dimension historischer Wirklichkeit kenntlich werden: die persönliche Entscheidung in ihrem Verhältnis zu Klugheit und Gewissen, zum gesunden Menschenverstand ${ }^{(16)}$. Auch diese Art des Persönlichen verzeichnet das Stichwort der Erinnerungskultur, denn die persönliche Entscheidung schreibt nicht Menschlichkeitsgeschichte, so wie die historische Persönlichkeit in Politik, Kultur und Technik Geschichte schreibt, sondern sie wird als Erinnerung festgehalten und weitergegeben, eben erinnert. So verstanden markiert der Begriff der Erinnerung möglicherweise auch eine Differenz zum Schreiben als Darstellungs- oder gar Bewegungsform, als Möglichkeitsbedingung von Geschichte ${ }^{(17)}$.

Propyläen, 1988; Jürgen KockA, „Ein chronologischer Bandwurm. Die Dauerausstellung des Deutschen Historischen Museums“, Geschichte und Gesellschaft, 32 (2006), S. 398-411.

14 Hierzu grundlegend: Thomas NipperdeY, „Nationalidee und Nationaldenkmal in Deutschland im 19. Jahrhundert“, Historische Zeitschrift, 206 (1968), S. 529-585.

15 Vgl. Daniel Levy, Natan Sznaider, Erinnerung im globalen Zeitalter. Der Holocaust, Frankfurt am Main, Suhrkamp, 2001.

16 Vgl. Wolfgang Hardtwig, „Von der, Vergangenheitsbewältigung zur Erinnerungskultur. Vom Umgang mit der NS-Vergangenheit in Deutschland“, in: Thomas Hertfelder, Andreas Rödder (Hg.), Modell Deutschland. Erfolgsgeschichte oder Illusion?, Göttingen, Vandenhoeck und Ruprecht, 2007, S. 171-189, hier S. 187.

17 In diesem Sinne Vilém Flusser, Die Schrift, Frankfurt am Main, Fischer, 1992, S. 11f.: „Die Schrift, dieses zeilenförmige Aneinanderreihen von Zeichen, macht überhaupt erst das Geschichtsbewußtsein möglich. [...] [V]or der Erfindung der Schrift ist nichts geschehen, alles hat sich nur ereignet. 
Wo nun Erinnerung Teil der nationalen Identität wird ${ }^{(18)}$, beginnt sich die Frage der Identität von Nationalgeschichten zu lösen, ohne dabei außerhalb historischer Wirklichkeit zu geraten. Das Stichwort der Erinnerungskultur verzeichnet schließlich auch die Integrationsschwierigkeiten für die politische Gemeinschaft, die sich daraus ergeben. Der Historiker Tony Judt etwa spricht mit Blick auf die ehemaligen kommunistischen Länder Osteuropas in diesem Zusammenhang von einer „Politik der bitteren Erinnerungen“ und einer „kompensatorischen Überfunktion des Gedächtnisses“, mit der nach 1989 eine Zeit „vorsätzlicher Amnesie“ zu Ende gehe ${ }^{(19)}$.

So komplex die Signatur der Erinnerung dem Begriffsgebrauch nach ist, schiebt sich mit ihr doch sehr deutlich die Frage nach dem Was der Geschichte in die Frage nach dem Wie von Geschichte. Wie ist ein Gedächtnis organisiert, mit dem das Persönliche und Individuelle und das Globale und Universelle Aspekte ein und desselben Zugriffs sind? Wie ist ein Gedächtnis organisiert, mit dem sich, möglicherweise nicht mehr in Form geschriebener Geschichte, eine andere historische Ereignishaftigkeit abbilden lässt, ein Gedächtnis, das quer steht zur Aufteilung in Alben privater Erinnerung einerseits und das nationale Schulbuch-Gedächtnis andererseits?

Nimmt man es wörtlich mit der Erinnerungskultur, dann ist hier vom Erinnern als einer Kulturtechnik die Rede. Die bloße Psychologie oder Anthropologie des Gedächtnisses steht damit grundsätzlich zur Diskussion. Während Modelle kultureller Repräsentation ein gewissermaßen „natürliches“ Erinnern voraussetzen, indem nach der immer nur „symbolischen Ausdehnung“ des „individuellen Erfahrungsgedächtnisses“(20) gefragt wird, und Literatur, Film, Denkmalskunst etc. als Medien eines voraus liegenden Gedächtnisses angesprochen werden, als Gedächtnismedien ${ }^{(21)}$, wird das Erinnern in der Perspektive der Kulturtechnik „aus dem anthropozentrischen Ursprung herausgerückt“ und in seiner historischen und materiellen Bedingtheit beschreibbar - als Realität eines Mediengedächtnisses ${ }^{(22)}$.

[...] Geschichte ist eine Funktion des Schreibens.“ Erinnern verweist in diesem Sinne auch auf den Untertitel des Buchs: Hat Schreiben Zukunft?

18 Vgl. etwa die Rede des ehemaligen deutschen Bundeskanzlers Gerhard Schröder zur Gedenkveranstaltung des Internationalen Auschwitz Komitees aus Anlass des 60. Jahrestages der Befreiung des Konzentrationslagers Auschwitz am 25. Januar 2005 in Berlin. URL: http://archiv.bundesregierung. de/Content/DE/Archiv16/Artikel/2005/01/2005-01-25--die-nazi-ideologie-war-menschengewolltund-menschengemacht-.html

19 Tony Judt, Geschichte Europas von 1945 bis zur Gegenwart, Franfurt am Main, Fischer, 2009, S. 959, hier S. 965.

20 A. Assman, Der lange Schatten der Vergangenheit (Anm. 11), S. 210.

21 Literatur als Gedächtnismedium bei Astrid ERLL, Gedächtnisromane: Literatur über den Ersten Weltkrieg als Medium englischer und deutscher Erinnerungskulturen in den 1920er Jahren, Trier, Wissenschaftlicher Verlag, 2003.

22 Bernhard Siegert, hier im Bezug auf die Kulturtechnik des Entwerfens: „Weiße Flecken und finstre Herzen. Von der symbolischen Weltordnung zur Weltentwurfsordnung“, in: Daniel Gethmann, Susanne Hauser (Hg.), Kulturtechnik Entwerfen. Praktiken, Konzepte und Medien in Architektur und Design Science, Bielefeld, Transcript, 2009, S. 19-47, hier S. 21. Vgl. im Bezug aufs Erinnern bereits Friedrich Kittler, „Vergessen“, in: Ulrich Nassen (Hg.), Texthermeneutik. Aktualität, Geschichte, Kritik, Paderborn, München, Schöningh, 1979, S. 195-221, hier S. 197: „Die Frage ist, wo und wie die Gedächtnisse funktionieren, die die Philosophie ,dem Menschen` zuschrieb.“ 
Es sind, wenn man so will, für die Gegenwart vor allem elektronische Bedingtheiten, die auf den Wandel der historischen Ereignishaftigkeit nach 1945 treffen. Im elektronischen Mediengedächtnis realisiert sich ein globaler Zugriff etwa auf Zeugnisse individuellen Handelns, aufs Persönliche also, ohne dass dieses bereits eingeschrieben wäre in eine Geschichte. Und der persönliche Zugriff auf dieses Gedächtnis ist nicht an die historisch-politischen Grenzen eines nationalen Interessenraums gebunden. Das Gedächtnis entspricht also weder der Verkörperung einer individuellen Erfahrung als historische Erfahrung ${ }^{(23)}$ noch der Aktualisierung eines offiziellen Geschichtsbilds. Es handelt sich gewissermaßen um ein ganz und gar flaches Gedächtnis, aus dem sich Geschichte allererst als eine Verknüpfung abhebt. Genau auf diesen Umstand scheint eine Installation wie die des Jahres 2011 im Deutschen Historischen Museum bezogen. Spiegel-Covers aus fünf Jahrzehnten, die in ihrer jeweiligen Aktualität einmal Politik und Zeitgeschichte kommentierten, geraten in eine neue, tatsächlich flache Anordnung. Während die Installation eine Geschichte zeigt, in der Hitler als ständiger Bezugspunkt des Redens und Schreiben über deutsche Vergangenheit und Gegenwart deutlich wird, stellt sich in ihr zugleich ein Effekt ein, der eben auch spezifisch scheint für das skizzierte Mediengedächtnis. In dem Maße, wie der Ausgangspunkt der Verknüpfungen, in diesem Falle ganz einfach die „Hitler-Hitler-Hitler..."-Reihe, sich selbst in die Fläche auflöst und, ganz wie beim Memory-Effekt, ihre Dauer annimmt, wandelt sich die Geschichte in ein Geisterbild.

Ein Film wie Der Untergang begibt sich im historischen Sujet direkt zurück auf seine eigene Bedingung - diejenige nämlich, unter der sich die historische Ereignishaftigkeit zu verändern beginnt. Scheint es doch, besonders am tragenden Schauspiel, offensichtlich, dass das ästhetische Konzept hier auf die Modellierung des Persönlichen zielt. Doch realisiert sich darin gerade keine auslegende Verknüpfung, durch die auch die Dimension einer Menschlichkeitsgeschichte in die Bildfläche treten würde - jene Dimension, in der der Holocaust als historisches Ereignis aufscheinen kann, und die damit aber ein historischen Urteilenkönnen nach 1945 überhaupt ermöglicht. An die Stelle des auslegenden Bezugs auf diesen Urteilshorizont tritt ganz einfach die Maßgabe der Authentizität. Unter den Bedingungen des Mediengedächtnisses aber, das nichts verkörpert, weder Korpus individueller Erfahrung noch eines nationalen Geschichtsbilds ist, kann Authentizität sich nicht mehr als Nachbildung verwirklichen. Vielmehr bedeutet es genau das, was sich am Film beobachten ließ: dass die Modellierung des Persönlichen immer nur an die schiere Ausdehnung des Gedächtnisses, an ein virtuelles Feld verweist.

Das Ausdruckgefüge, das der Der Untergang in seinem filmischen Verlauf zusammensetzt, realisiert sich im Zuschauerwahrnehmen als Gestalt eines persönlichen Empfindens, das, streng genommen, keinerlei historischen Sinn hat. Es realisiert sich als Geistererscheinung einer im Nirgendwo des Gedächtnisses gebunkerten Liebe

23 Einen Unterschied zur Aufhebung der Erfahrung in Geschichte markiert der Begriff der Zeugenschaft. Vgl. Annette Wieviorka, L’ère du témoin, Paris, Hachette, 2002. Die „Ära des Zeugen“, die Zeitzeugengeschichte steht in engem Zusammenhang mit der Entwicklung des Mediengedächtnisses. Zeugnisablegen hat auch einen technischen Sinn. Großprojekte der Oral History, wie beispielsweise Steven Spielbergs Visual History Archive, stellen eine Herausforderung an Speicher- und Verarbeitungskapazitäten dar, sie erzeugen gleichsam erhöhte Gedächtnisleistungen technischer Medien. 
und Verzweiflung. Damit bildet es eine weder fiktive noch bezeugende, sondern eine künstliche Erinnerung, in der, allem Anschein zum Trotz, keine Antworten auf die Fragen der historischen Identität verborgen liegen.

\title{
Zusammenfassung
}

Untersucht wird, wie die Wiederholung des Bildes Adolf Hitlers seit 1945 eine Dauer erzeugt, die sich vom historischen Bild oder Dokument ebenso ablöst wie von bestimmten aktualisierenden Ikonografien. Am Beispiel des Films Der Untergang (D 2004, Oliver Hirschbiegel) wird gezeigt, wie beispielsweise der „Untergang des Tausendjährigen Reichs" in der Dauer des Hitler-Bilds als etwas scheinbar Zeitloses gegen die Verlaufsform von Geschichte insistiert. Der Untergang gleicht darin einem Phantasma, das seine Zeit in einer Art Geisterbild „Hitler" hat. Im Film lässt sich dies als audiovisuelle Gestalt einer geisterhaften Erinnerung analytisch greifbar machen. Der Aufsatz schlägt vor, diesen Zusammenhang mit dem Begriff des Memory-Effekts zu beschreiben.

\begin{abstract}
The paper examines how the repetition of the image of Adolf Hitler since 1945 produces a duration that differs from the historic image or document as well as from certain politically updated iconographies. Using the example of the movie Downfall ( $D$ 2004, Oliver Hirschbiegel) it will be shown that, for example, the "downfall of the Third Reich» through the duration of the Hitler-image insists as something apparently timeless against historical mutability. The Downfall seems like a phantasm that has its time in the ghost image "Hitler.» In the film, this will be made analytically tangible as the audiovisual shape of a ghostly memory. The paper proposes to describe this relationship by the concept of memory effect.
\end{abstract}

